\title{
Adiponectin Is Negatively Associated With Disease Activity and Sharp Score in Rheumatoid Arthritis: a Cross-sectional Study on Treatment-naïve Han Chinese Patients
}

\section{Xixi Chen}

Sichuan Provincial People's Hospital, University of Electronic Science and Technology of China

\section{Kaiwen Wang}

University of Cambridge

Tao Lu

Sichuan Provincial People's Hospital, University of Electronic Science and Technology of China Jiajia Wang

Sichuan Provincial People's Hospital, University of Electronic Science and Technology of China Ting Zhou

Sichuan Provincial People's Hospital, University of Electronic Science and Technology of China Juan Tian

The First People's Hospital of Tianmen City

\section{Bin Zhou}

Sichuan Provincial People's Hospital, University of Electronic Science and Technology of China

\section{Li Long}

Sichuan Provincial People's Hospital, University of Electronic Science and Technology of China Qiao Zhou ( 22922934@qq.com )

Sichuan Provincial People's Hospital, University of Electronic Science and Technology of China

\section{Research Article}

Keywords: Rheumatoid arthritis, adiponectin, DAS28(ESR), Sharp score, cross-sectional study

Posted Date: November 1st, 2021

DOl: https://doi.org/10.21203/rs.3.rs-1020176/v1

License: (c) (1) This work is licensed under a Creative Commons Attribution 4.0 International License. Read Full License 


\section{Abstract}

Background: The association and potential role of the protein hormone adiponectin in autoimmune diseases causing musculoskeletal disorders, including rheumatoid arthritis (RA), are controversial. Conflicting results may arise from the influences of confounding factors linked to genetic backgrounds, disease stage, disease-modifying anti-rheumatic drugs and patients' metabolic characteristics. Here, we examined serum level of adiponectin and its relationship with disease activity score 28 with erythrocytes sedimentation rate (DAS28[ESR]) and Sharp score in a treatment-naïve Han Chinese RA population.

Methods: This cross-sectional study enrolled 125 RA patients. Serum level of total adiponectin was assessed by enzyme-linked immunosorbent assay (ELISA). Other important clinical and laboratory parameters were collected from the hospital database. DAS28(ESR) was calculated according to the equation previously published. Sharp score was evaluated based on hands radiographs by an independent radiologist. The correlation between serum adiponectin level and DAS28(ESR) or the Sharp score was investigated by univariable and multivariable regression analyses. Multiple imputation by chained equations was used to account for missing data.

Results: Univariable analyses showed significant positive correlation between DAS28(ESR) and age or CReactive Protein (CRP) (both $p=0.003$ ), while serum adiponectin level was negatively correlated with DAS28(ESR) ( $p=0.015)$. The negative correlation between adiponectin level and DAS28(ESR) remained true in multivariable analyses adjusted for confounders. In addition, the univariable analyses revealed positively correlations of Sharp score to disease duration $(p<0.001), \operatorname{CRP}(p=0.023)$ and ESR $(p<$ 0.001). In the multivariable model adjusted for confounders, adiponectin was negatively correlated with Sharp score $(p=0.044)$.

Conclusion: In this single-institution cross-sectional study, serum adiponectin level in treatment-naive RA patients is negatively correlated with DAS28(ESR) and the Sharp score after adjustment for prominent identified confounders. Serum adiponectin may be potentially useful for assessing disease activity and radiographic progression of $\mathrm{RA}$.

\section{Background}

Rheumatoid arthritis (RA) is a chronic inflammatory-related autoimmune disease that primarily affects joints and causes musculoskeletal disorders [1]. The characteristics of RA include synovium hyperplasia, lymphocyte infiltration and abnormal proliferation of fibroblast-like synoviocytes, all of which leading eventually to erosive joint destruction [2].

Adiponectin is a hormone protein mainly secreted by adipose tissue but also by various other cells, including skeletal myocytes and cardiomyocytes [3]. It is abundantly present in the circulation, accounting for $0.01 \%$ of total plasma proteins [4]. The potential role of adiponectin in RA has been actively investigated. Conflicting data on its role in RA have been reported. Adiponectin could act as proinflammatory mediator as its serum level positively correlates with disease activity [5-9] or 
radiographic progression [10]. However, this association has not been unanimously agreed upon, with opposite results being reported from other studies [11-14]. Given these heterogenous findings and since most of these studies were conducted in Caucasian patients or with relatively higher body mass index (BMI) [15-17] and some study did not adjust for confounders [10], we conducted a cross-sectional study to evaluate the relationship between adiponectin and disease activity as well as radiography outcomes in a cohort of treatment-naïve Chinese RA patients using both univariable and multivariable methods.

\section{Materials And Methods}

\section{Study population}

Between 2012 and 2020, one hundred twenty-five patients with rheumatoid arthritis according to the American College of Rheumatology 1987 criteria were included in this study. Patients with co-morbidities such as hypertension, diabetes, hypercholesterolaemia, chronic inflammatory disease, autoimmune disease, or cancer were excluded. At the time of the study, none of the patients had received treatment against RA. All patients belonged to the Han ethnic group. In addition to the RA patients, 34 healthy participants were studied to evaluate the baseline level of total serum adiponectin in the general population. The study was conducted in accordance with the Declaration of Helsinki and was approved by the Ethical Committee of Sichuan Provincial People's Hospital. All subjects signed informed consent.

\section{Clinical and laboratory measurements}

Clinical information and laboratory data were obtained through a detailed interview, self-reported questionnaires, physical examination, and blood tests. The BMI was calculated as [body weight/height ${ }^{2}$ ] $\left(\mathrm{kg} / \mathrm{m}^{2}\right)$. Disease activity was measured using modified disease activity score 28 with erythrocyte sedimentation rate (DAS28[ESR]) [18].

The blood of all subjects was collected in the morning, after overnight fasting. Levels of CRP, ESR, rheumatoid factor (RF) and anti-cyclic citrullinated peptide (CCP) antibodies were measured by the Clinical Laboratory of Sichuan Provincial People's Hospital. Serum concentrations of total adiponectin were measured by enzyme-linked immunosorbent assay (ELISA), using the kit purchased from BioVision, USA. Samples were prepared at appropriate dilutions and assayed according to the manufacturer's protocol.

\section{Radiographic outcomes}

Single-view anterior-posterior $\mathrm{x}$-rays of both hands were scored using the van der Heijde modification of the Sharp method (SHS) (referred to as Sharp score) by a single experienced reader blinded to patient characteristics [19].

\section{Statistical analysis}


Continuous variables are expressed as means \pm standard deviations, and categorical data are expressed as a number (percentage). Statistical significance was calculated using $t$ test (normal distribution) or Kruskal-Wallis test (skewed distribution) unless stated otherwise. A univariable analysis model was used to determine the significance of the association between all clinical variables and the outcome, DAS28(ESR), or Sharp score. Next, a multivariable linear regression model was used to examine independent associations between serum adiponectin level and each of the outcome, after adjusting for confounders. Variables were selected as confounders if they were either significantly associated with the outcome, or if when added to the basic model or removed from the full model, a change in effect estimate of adiponectin level by more than $10 \%$ was observed. 'Basic model' refers to the unadjusted univariable regression model between adiponectin and the outcome, whereas 'full model' refers to multivariable regression model adjusting for all covariates. Swollen joint count (SJC), tender joint count (TJC) and ESR were excluded from the full model of DAS28(ESR) because they were already captured by the score.

Missing data were noticed in age, disease duration, height, weight, ESR, CRP, RF, CCP, SJC, TJC, Sharp score and adiponectin. To maximise statistical power and minimise potential bias, we used multiple imputation by chained equations to create five imputed datasets to account for missing data [20]. Analyses were repeated on each of the imputed datasets and final results were obtained by combining the results from each individual analysis according to Rubin's rules [21]. In addition, sensitivity analysis was performed to identify whether the created complete data had a significant difference from preimputation data. All the analyses were performed with R software Version 3.4.3 (http://www.Rproject.org) and EmpowerStats (http://www.empowerstats.com, X\&Y Solutions, Inc., Boston, MA). A P value less than 0.05 was considered statistically significant.

\section{Results}

\section{Clinical and laboratory characteristics of the patients}

The characteristics of the healthy controls are summarised in Table 1. The clinical and laboratory profiles of the RA patients are summarised in Table 2. A total of 125 RA patients with a mean age of $55.7 \pm 12.4$ years were included, of which $94(75.2 \%)$ were female. Compared to male patients, female patients were significantly younger ( $54.4 \pm 12.7$ vs $59.9 \pm 10.1, p=0.033)$. The average height and weight also showed significant differences between genders (both with $p<0.001$ ), although the two groups had a compatible average BMI, which was overall of $22.6 \pm 3$.7. The mean DAS28(ESR) of the whole cohort was $5.4 \pm 3.3$ and the mean value of serum total adiponectin was $25.0 \pm 19.1 \mu \mathrm{g} / \mathrm{mL}$. Serum total adiponectin in an age- and sex-matched healthy population sample was significantly lower $(13.6 \pm 5.5 \mu \mathrm{g} / \mathrm{mL} ; \mathrm{p}=0.015$ compared with the RA group). 
Table 1

Clinical and laboratory characteristics of the enrolled healthy controls

\begin{tabular}{|lllll|}
\hline Characteristic & Male & Female & Total & P value \\
\hline $\mathrm{N}(\%)$ & $9(26.4)$ & $25(73.5)$ & $34(100)$ & \\
\hline Age (years) & $59.1 \pm 10.5$ & $53.5 \pm 9.3$ & $55.1 \pm 9.9$ & $\mathbf{0 . 0 1 6}$ \\
\hline Height $(\mathrm{cm})$ & $170.7 \pm 8.1$ & $156.5 \pm 5.3$ & $159.8 \pm 4.3$ & $<0.001$ \\
\hline Weight $(\mathrm{kg})$ & $67.3 \pm 7.9$ & $55.9 \pm 8.6$ & $58.9 \pm 12.1$ & $<0.001$ \\
\hline BMI $\left(\mathrm{kg} / \mathrm{m}^{2}\right)$ & $24.4 \pm 1.3$ & $22.9 \pm 4.0$ & $23.7 \pm 2.9$ & 0.099 \\
\hline Adiponectin $(\mu \mathrm{g} / \mathrm{mL})$ & $12.5 \pm 4.0$ & $15.2 \pm 6.2$ & $13.6 \pm 5.5$ & 0.058 \\
\hline
\end{tabular}


Table 2

Clinical and laboratory characteristics of the enrolled patients

\begin{tabular}{|llllll|}
\hline Characteristic & N (\%) & Male & Female & Total & P value \\
\hline Gender (N, \%) & $125(100)$ & $31(24.8)$ & $94(75.2)$ & & \\
\hline Age (years) & $124(99.2)$ & $60.9 \pm 11.4$ & $54.0 \pm 12.3$ & $55.7 \pm 12.4$ & 0.007 \\
\hline Height (cm) & $122(97.6)$ & $167.2 \pm 8.2$ & $155.5 \pm 5.0$ & $158.4 \pm 7.8$ & $<0.001$ \\
\hline Weight (kg) & $122(97.6)$ & $64.3 \pm 11.0$ & $54.4 \pm 10.3$ & $56.8 \pm 11.3$ & $<0.001$ \\
\hline BMI (kg/m $\left.{ }^{2}\right)$ & $122(97.6)$ & $23.0 \pm 3.2$ & $22.4 \pm 3.8$ & $22.6 \pm 3.7$ & 0.504 \\
\hline $\begin{array}{l}\text { Disease duration } \\
\text { (month) }\end{array}$ & $118(94.4)$ & $105.2 \pm 116.4$ & $109.0 \pm 107.3$ & $108.0 \pm 109.1$ & 0.871 \\
\hline SJC & $123(98.4)$ & $4.5 \pm 6.0$ & $3.8 \pm 6.3$ & $4.0 \pm 6.2$ & 0.637 \\
\hline TJC & $123(98.4)$ & $6.8 \pm 8.7$ & $6.9 \pm 9.0$ & $6.9 \pm 8.9$ & 0.844 \\
\hline CRP (mg/dL) & $91(72.8)$ & $33.9 \pm 38.3$ & $40.1 \pm 57.3$ & $38.5 \pm 52.8$ & 0.627 \\
\hline ESR (mm/H) & $124(99.2)$ & $52.8 \pm 30.9$ & $56.2 \pm 33.0$ & $55.4 \pm 32.4$ & 0.618 \\
\hline DAS28(ESR) & $123(98.4)$ & $5.7 \pm 3.4$ & $5.3 \pm 3.3$ & $5.4 \pm 3.3$ & 0.594 \\
\hline RF (IU/mL) & $122(97.6)$ & $444.2 \pm 685.0$ & $321.3 \pm 469.3$ & $352.5 \pm 532.0$ & 0.269 \\
\hline Anti-CCP (Ru/mL) & $119(95.2)$ & $242.5 \pm 173.1$ & $230.8 \pm 170.4$ & $233.7 \pm 170.4$ & 0.746 \\
\hline Adiponectin ( $\mu$ g /mL) & $118(94.4)$ & $21.4 \pm 20.3$ & $26.3 \pm 18.6$ & $25.0 \pm 19.1$ & 0.223 \\
\hline Sharp score & $115(92.0)$ & $43.1 \pm 63.5$ & $44.3 \pm 51.7$ & $44.0 \pm 54.7$ & 0.917 \\
\hline $\begin{array}{l}\text { Abbreviations: BMl: body mass index; CCP: cyclic citrullinated peptides; CRP: C-reactive protein; } \\
\text { DAS28: disease activity score of 28 joints; ESR: erythrocyte sedimentation rate; RF: rheumatoid factor; } \\
\text { SJC: swollen joint count; TJC: tender joint count. Continuous variables are expressed as means } \pm \\
\text { standard deviations and categorical data using number (percentage). }\end{array}$ & & \\
\hline
\end{tabular}

\section{Identification of factors correlated with DAS28(ESR) and Sharp score by univariable analysis}

The relationships between different clinical parameters and disease activity measured by DAS28(ESR) were assessed by univariable linear regression analysis. Analyses using the univariable model against DAS28(ESR) indicated significant positive correlations with age and CRP $(p=0.0026$ and $p=0.003$, respectively; Table 3$)$. In contrast, serum adiponectin was negatively correlated with DAS28(ESR) ( $p=$ 0.015). This model did not detect any further significant association between DAS28(ESR) and other variables $(p>0.05)($ Table 3$)$. 
Table 3

Univariable regression results against DAS28(ESR) or Sharp score

\begin{tabular}{|c|c|c|c|c|}
\hline \multirow[t]{2}{*}{ Variables } & \multicolumn{2}{|l|}{ DAS28(ESR) } & \multicolumn{2}{|l|}{ Sharp score } \\
\hline & $\beta /$ Odds ratio $(95 \% \mathrm{Cl})$ & $\mathbf{P}$ & $\beta /$ Odds ratio $(95 \% \mathrm{Cl})$ & $\mathbf{P}$ \\
\hline \multicolumn{5}{|l|}{ GENDER } \\
\hline Male & 0 & & 0 & \\
\hline Female & $-0.27(-1.62,1.09)$ & 0.698 & $1.22(-21.65,24.09)$ & 0.917 \\
\hline Age & $0.07(0.03,0.12)$ & 0.003 & $0.62(-0.17,1.41)$ & 0.128 \\
\hline Disease duration & $0.00(-0.00,0.01)$ & 0.177 & $0.30(0.22,0.38)$ & $<0.001$ \\
\hline Height & $-0.03(-0.10,0.05)$ & 0.497 & $-0.26(-1.54,1.01)$ & 0.688 \\
\hline Weight & $-0.03(-0.08,0.03)$ & 0.331 & $-0.68(-1.58,0.22)$ & 0.141 \\
\hline BMI & $-0.06(-0.23,0.10)$ & 0.444 & $-2.23(-5.03,0.56)$ & 0.121 \\
\hline RF & $-0.00(-0.00,0.00)$ & 0.886 & $0.01(-0.01,0.03)$ & 0.311 \\
\hline $\mathrm{CCP}$ & $-0.00(-0.01,0.00)$ & 0.204 & $-0.01(-0.07,0.03)$ & 0.853 \\
\hline CRP & $0.02(0.01,0.03)$ & 0.003 & $0.26(0.04,0.049)$ & 0.023 \\
\hline ESR & $\mathrm{N} / \mathrm{A}$ & $\mathrm{N} / \mathrm{A}$ & $0.65(0.36,0.94)$ & $<0.001$ \\
\hline SJC & $\mathrm{N} / \mathrm{A}$ & $\mathrm{N} / \mathrm{A}$ & $2.05(0.51,3.59)$ & 0.010 \\
\hline TJC & $\mathrm{N} / \mathrm{A}$ & $\mathrm{N} / \mathrm{A}$ & $2.07(1.02,3.13)$ & $<0.001$ \\
\hline Adiponectin & $-0.04(-0.07,-0.01)$ & 0.015 & $-0.39(-0.99,0.20)$ & 0.198 \\
\hline
\end{tabular}

Analyses using the univariable model against the Sharp score revealed positive correlations with disease duration, CRP, ESR, SJC and TJC ( $p<0.001, p=0.023, p<0.001, p=0.010$ and $p<0.001$, respectively), whereas no correlation was observed with serum adiponectin level (Table 3 ).

\section{Analyses by multivariable linear regression shows an independent relationship between adiponectin level and DAS28(ESR)}

We further explored the relationship between adiponectin level and the outcomes using multivariable linear regression analyses adjusted for confounders. Age, BMI and CRP were finally selected as confounders for DAS28(ESR). Age, BMI, disease duration, CRP, ESR, SJC and TJC were finally selected as confounders for Sharp score. 
In the crude models, there was no adjustment for confounders, while in model I, two confounders were adjusted when using DAS28(ESR) and three confounders were adjusted when using the Sharp score. Model II was adjusted for all confounders. DAS28(ESR) and adiponectin were significantly and negatively correlated in all three models (Table 4). When stratified by gender, the same trend existed in female but not male patients. In the models against the Sharp score, after adjusting for confounders, there was a negative association between adiponectin level and Sharp score. In female patients, the same trend also existed (Table 4). The scatter plots of the linear regression obtained after adjusting for all confounders are shown in Figure 1.

Table 4

Multivariable linear relationship stratified by gender, $\beta(95 \% \mathrm{Cl})$ of adiponectin $(\mu \mathrm{g} / \mathrm{mL})$

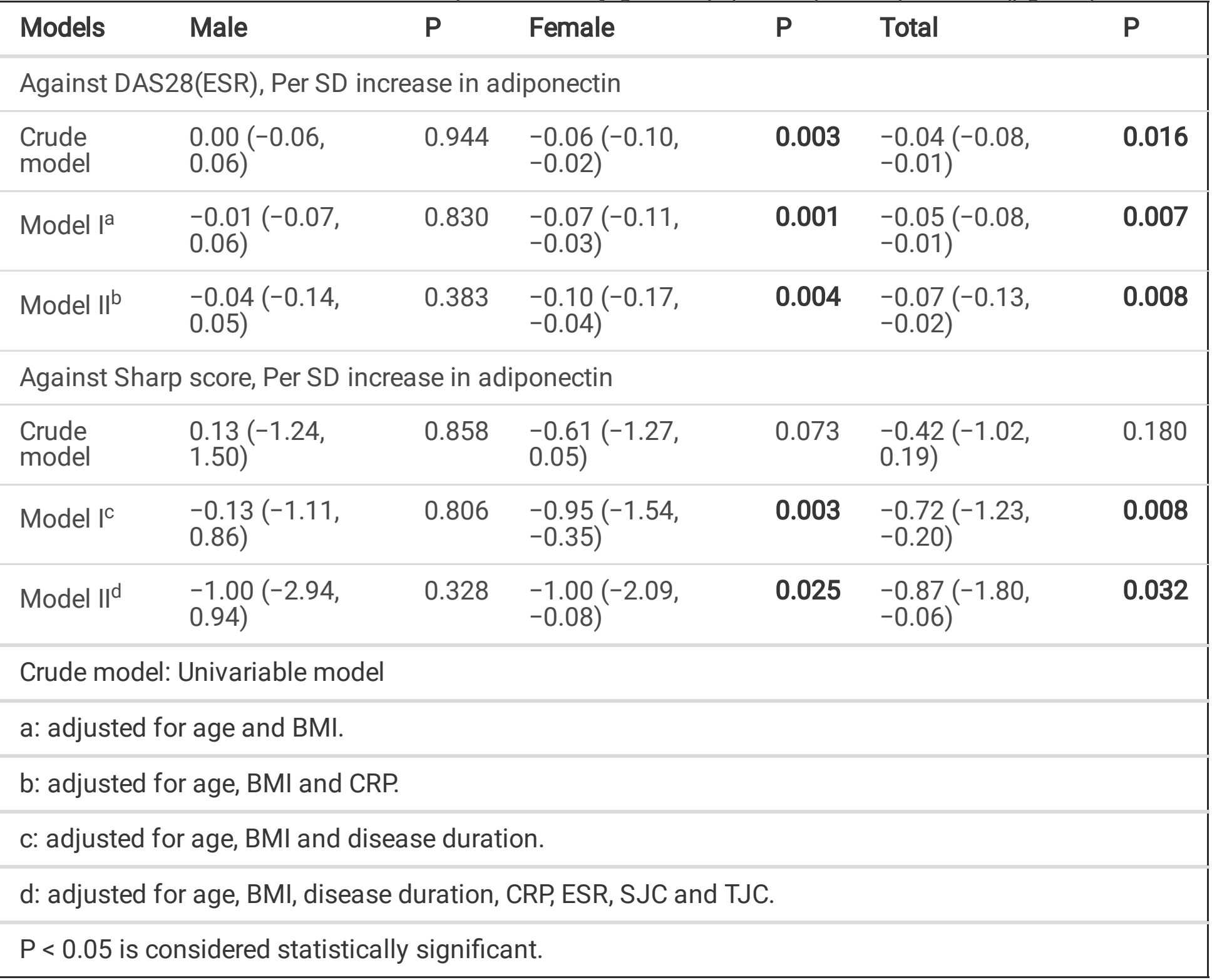

Exploration of modifier and interaction effects on adiponectin/DAS28(ESR) or adiponectin/Sharp score associations 
We explored potential modifier or interaction effects from age, BMI and CRP, and did not find any potential factor interfering with adiponectin/DAS28(ESR) association. The same analysis was performed for adiponectin/Sharp score association, with the addition of the variables 'disease duration', 'ESR', 'SJC' and 'TJC'. Similarly, no potential modifier or interaction factor was found.

\section{Sensitivity analysis}

The amount of missing data for the different variables ranged from 0 to $27 \%$ (Table 2). Eighty-four out of the 125 (67\%) patients had complete data for all variables for the main analyses. The distributions of the original and imputed variables are depicted in Supplementary Table S1 (Additional file 1). Regression analyses using multiple imputed datasets gave similar results to those undertaken on the original datasets, as displayed in Supplementary Table S2 (Additional file 1).

\section{Discussion}

To evaluate the relationships between serum adiponectin and RA disease activity, we performed a crosssectional investigation on a Chinese RA population. After accounting for confounders, multivariable regression analyses showed a negative correlation between serum adiponectin level and DAS28(ESR) or Sharp score. To avoid bias from missing data, we carried out a sensitivity analysis using full imputed data. With this sensitivity analysis, the associations of DAS28(ESR) and Sharp score with serum adiponectin remained statistically significant. These results are in keeping with previous studies showing that serum adiponectin level negatively correlated with disease activity in RA $[15,22]$.

In vitro experiments with RA synovial fibroblasts indicated that adiponectin significantly inhibits IL-1induced RA synovial fibroblasts proliferation [23]. In a DBA/1 mouse model of collagen-induced arthritis, adiponectin treatment significantly mitigated the severity of arthritis along with a decrease in the expression of TNF-a, IL-1 and MMP-3 in joint tissues [24]. Anti-TNF treatment in female patients with RA was associated with increased adiponectin levels, which may dampen the systemic inflammatory response associated with RA [25]. These findings support an anti-inflammatory role for adiponectin in RA.

However, several studies, including a metanalysis [16], led to the conclusion that there was either no or a positive correlation between adiponectin level and inflammatory markers such as chemokines, CRP or DAS28 $[5,6,26,27]$. This inconsistency may stem from the study designs and sampled populations, but also indicates that adiponectin may play different roles in inflammation, depending on disease characteristics, comorbidities, treatment, genetic backgrounds and physiological specifics of the patients.

For instance, when our analysis of the correlation between disease activity score and adiponectin level was stratified by gender, the negative correlation observed in the total and female RA populations did not verify in male patients. This result might be due to the smaller sample size of the male compared with the female group. Alternatively, this result could indicate that adiponectin has different implications in male and female patients. Thus, male/female ratio may influence the outcome of studies analysing the correlation between serum adiponectin and inflammatory markers, and cause part of the heterogeneity 
reported in the literature. This hypothesis is supported by a study from Li et al. [4], based on a female RA population, which also demonstrated clear negative correlations between serum adiponectin levels and DAS28(ESR). Further, the recent discovery of an adiponectin genetic variant associated with anti-CCP antibodies in RA female patients suggests a differential significance of adiponectin between sexes [28].

Another specificity of our study is that the enrolled patients had relatively high DAS28(ESR) and had not received any treatment for RA. Different treatments may affect the level of adiponectin differentially [3, $25,26,29,30]$. Therefore, the absence of potential interferences between disease-modifying drugs and adiponectin level may have facilitated the demonstration of negative correlations between adiponectin level and DAS28(ESR) or Sharp score. Similarly, the relative homogeneity of our cohort regarding its ethnicity may have diminished the influence of potential confounding factors such as genetic background, BMI [31,32] and adiponectin variants [28] on the association between the adiponectin level and disease activity [7].

Finally, some limitations and specificities should be taken into consideration when interpreting the data from our study. First, this is a cross-sectional clinical study without longitudinal data, and our results do not imply causation. Second, the participants were restricted to Chinese ethnicity from one district and predominantly females. It is known that Chinese populations have lower average BMI compared to other populations. Finally, as discussed above, there might be unconsidered covariates affecting RA severity or serum adiponectin such as genetic variants (i.e., RA risk HLA alleles and small nucleotide polymorphisms [SNPs] in the adiponectin gene) or adiponectin isoforms [7]. Further molecular investigation involving in vitro experiments and genetic comparisons in different populations are needed to untangle the pathophysiological role of adiponectin in RA progression.

In conclusion, multiple regression analysis showed a negative and independent correlation between serum adiponectin and DAS28(ESR) or Sharp score. Therefore, measurement of serum adiponectin may be potentially useful for assessing disease activity of RA in Chinese patients regardless of ongoing medications.

\section{Abbreviations}

BMI: body mass index

CCP: cyclic citrullinated peptide

CRP: C-Reactive Protein

DAS28(ESR): disease activity score 28 with erythrocyte sedimentation rate

ELISA: enzyme-linked immunosorbent assay

ESR: erythrocyte sedimentation rate 
RA: rheumatoid arthritis

RF: rheumatoid factor

SJC: swollen joint count

SNPs: small nucleotide polymorphisms

TJC: tender joint count

\section{Declarations}

\section{Ethics approval and consent to participate}

This study was approved by the Ethical Committee of Sichuan Provincial People's Hospital. All subjects signed informed consent.

\section{Consent for publication}

All patients signed a consent for publication form.

\section{Availability of data and materials}

The datasets used and/or analysed in the current study are available from the corresponding author upon reasonable request.

\section{Competing interests}

The authors declare that they have no competing interests.

\section{Funding}

This research was supported by the Foundation for Outstanding Young Talents, Science and Technology Department of Sichuan Province (2020JDJQ0067).

\section{Authors' contributions}

$X C, B Z, L L$ and QZ contributed to the conception and design of the work. XC, TL, JW, TZ and JT contributed to data collection. KW and QZ analysed and interpreted the data. XC and KW drafted the manuscript. LL and QZ substantively revised the manuscript. All authors read and approved the final manuscript.

\section{Acknowledgements}

Not applicable 


\section{References}

1. Mclnnes, I.B. and G. Schett, The pathogenesis of rheumatoid arthritis. N Engl J Med, 2011. 365(23): p. 2205-19.

2. Cooles, F.A. and J.D. Isaacs, Pathophysiology of rheumatoid arthritis. Curr Opin Rheumatol, 2011. 23(3): p. 233-40.

3. Brezovec, N., et al., Adiponectin Deregulation in Systemic Autoimmune Rheumatic Diseases. International Journal of Molecular Sciences, 2021. 22(8): p. 4095.

4. Li, P., et al., Low-molecular-weight adiponectin is more closely associated with disease activity of rheumatoid arthritis than other adiponectin multimeric forms. Clin Rheumatol, 2015. 34(6): p. 102530.

5. Ebina, K., et al., Serum adiponectin concentrations correlate with severity of rheumatoid arthritis evaluated by extent of joint destruction. Clin Rheumatol, 2009. 28(4): p. 445-51.

6. Minamino, $\mathrm{H}_{\text {., }}$ et al., Increased circulating adiponectin is an independent disease activity marker in patients with rheumatoid arthritis: A cross-sectional study using the KURAMA database. PLoS One, 2020. 15(3): p. e0229998.

7. Lei, Y., et al., Association Between Adiponectin and Clinical Manifestations in Rheumatoid Arthritis. J Interferon Cytokine Res, 2020. 40(10): p. 501-508.

8. Khajoei, S., et al., Serum levels of adiponectin and vitamin D correlate with activity of Rheumatoid Arthritis. Molecular Biology Reports, 2019. 46(2): p. 2505-2512.

9. Otero, M., et al., Changes in plasma levels of fat-derived hormones adiponectin, leptin, resistin and visfatin in patients with rheumatoid arthritis. Ann Rheum Dis, 2006. 65(9): p. 1198-201.

10. Giles, J.T., D.M. van der Heijde, and J.M. Bathon, Association of circulating adiponectin levels with progression of radiographic joint destruction in rheumatoid arthritis. Ann Rheum Dis, 2011. 70(9): p. $1562-8$.

11. Yoshino, T., et al., Elevated serum levels of resistin, leptin, and adiponectin are associated with Creactive protein and also other clinical conditions in rheumatoid arthritis. Intern Med, 2011. 50(4): p. 269-75.

12. Rho, Y.H., et al., Adipocytokines are associated with radiographic joint damage in rheumatoid arthritis. Arthritis Rheum, 2009. 60(7): p. 1906-14.

13. Šenolt, L., et al., Increased adiponectin is negatively linked to the local inflammatory process in patients with rheumatoid arthritis. Cytokine, 2006. 35(5): p. 247-252.

14. Baker, J.F., et al., Insulin-like Growth Factor 1 and Adiponectin and Associations with Muscle Deficits, Disease Characteristics, and Treatments in Rheumatoid Arthritis. The Journal of Rheumatology, 2015: p. jrheum.150280.

15. Laurberg, T.B., et al., Plasma adiponectin in patients with active, early, and chronic rheumatoid arthritis who are steroid-and disease-modifying antirheumatic drug-naive compared with patients with osteoarthritis and controls. J Rheumatol, 2009. 36(9): p. 1885-91. 
16. Lee, Y.H. and S.C. Bae, Circulating adiponectin and visfatin levels in rheumatoid arthritis and their correlation with disease activity: A meta-analysis. Int J Rheum Dis, 2018. 21(3): p. 664-672.

17. Bustos Rivera-Bahena, C., et al., Peripheral blood leptin and resistin levels as clinical activity biomarkers in Mexican Rheumatoid Arthritis patients. Reumatol Clin, 2016. 12(6): p. 323-326.

18. Prevoo, M.L., et al., Modified disease activity scores that include twenty-eight-joint counts. Development and validation in a prospective longitudinal study of patients with rheumatoid arthritis. Arthritis Rheum, 1995. 38(1): p. 44-8.

19. van der Heijde, D., How to read radiographs according to the Sharp/van der Heijde method. J Rheumatol, 2000. 27(1): p. 261-3.

20. Clark, T.G. and D.G. Altman, Developing a prognostic model in the presence of missing data: an ovarian cancer case study. J Clin Epidemiol, 2003. 56(1): p. 28-37.

21. Donders, A.R., et al., Review: a gentle introduction to imputation of missing values. J Clin Epidemiol, 2006. 59(10): p. 1087-91.

22. Komai, N., et al., Anti-tumor necrosis factor therapy increases serum adiponectin levels with the improvement of endothelial dysfunction in patients with rheumatoid arthritis. Mod Rheumatol, 2007. 17(5): p. 385-90.

23. Liu, D., S. Luo, and Z. Li, Multifaceted roles of adiponectin in rheumatoid arthritis. Int Immunopharmacol, 2015. 28(2): p. 1084-90.

24. Lee, S.W., et al., Adiponectin mitigates the severity of arthritis in mice with collagen-induced arthritis. Scand J Rheumatol, 2008. 37(4): p. 260-8.

25. Serelis, J., et al., Effect of anti-TNF treatment on body composition and serum adiponectin levels of women with rheumatoid arthritis. Clin Rheumatol, 2008. 27(6): p. 795-7.

26. Härle, P., et al., No change of serum levels of leptin and adiponectin during anti-tumour necrosis factor antibody treatment with adalimumab in patients with rheumatoid arthritis. Ann Rheum Dis, 2006. 65(7): p. 970-1.

27. Vasileiadis, G.K., et al., Adipocytokines in Untreated Newly Diagnosed Rheumatoid Arthritis: Association with Circulating Chemokines and Markers of Inflammation. Biomolecules, 2021. 11(2): p. 325 .

28. Zhao, Y.L., et al., Association of adiponectin and adiponectin receptor gene polymorphisms with rheumatoid arthritis in a Chinese population. Postgrad Med J, 2020. 96(1133): p. 149-155.

29. Toussirot, E.B., D.; Gueugnon, C.; Dumoulin, G., Adiponectin in Autoimmune Diseases. Current Medicinal Chemistry, 2012. 19(32): p. 7.

30. Toussirot, E., et al., Increased high molecular weight adiponectin and lean mass during tocilizumab treatment in patients with rheumatoid arthritis: a 12-month multicentre study. Arthritis Research \& Therapy, 2020. 22(1): p. 224.

31. Zhang, Y., et al., Elevated adiponectin predicts the development of rheumatoid arthritis in subjects with obesity. Scandinavian Journal of Rheumatology, 2020. 49(6): p. 452-460. 
32. Chihara, K., et al., Re-evaluation of serum leptin and adiponectin concentrations normalized by body fat mass in patients with rheumatoid arthritis. Scientific Reports, 2020. 10(1): p. 15932.

\section{Figures}
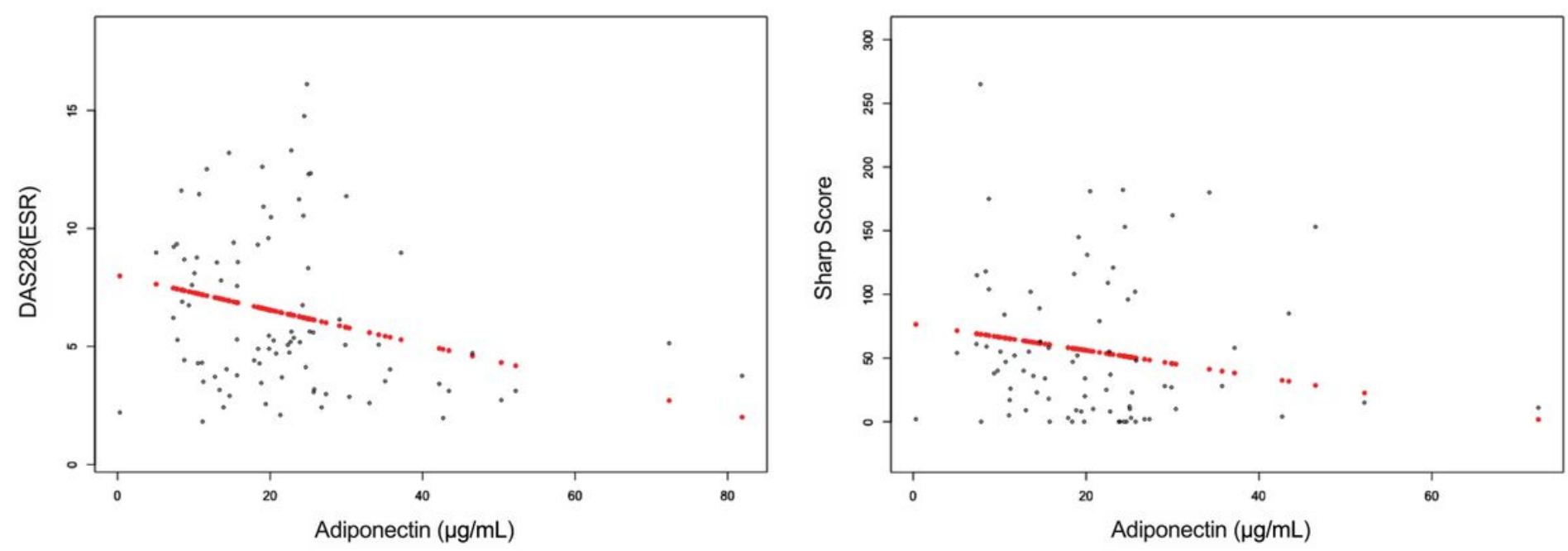

\section{Figure 1}

Scatter plots for serum adiponectin level versus DAS28(ESR) (left) or Sharp score (right). The linear regression shows a significantly negative association between serum adiponectin and the two outcome measures (red lines).

\section{Supplementary Files}

This is a list of supplementary files associated with this preprint. Click to download.

- additionalfile.docx 